CLINICAL STUDY

\title{
Impact of GH replacement therapy on sleep in adult patients with GH deficiency of pituitary origin
}

\author{
Lisa L Morselli ${ }^{1,2}$, Arlet Nedeltcheva ${ }^{1}$, Rachel Leproult ${ }^{1}$, Karine Spiegel ${ }^{3,6}$, Enio Martino ${ }^{2}$, Jean-Jacques Legros ${ }^{5}$, \\ Roy E Weiss ${ }^{1}$, Jean Mockel $^{4}$, Eve Van Cauter ${ }^{1}$ and Georges Copinschi ${ }^{3,4}$ \\ ${ }^{1}$ Department of Medicine, Sleep, Metabolism and Health Center (SMAHC), MC 1027, University of Chicago, Chicago, Illinois, 60637 USA, ${ }^{2}$ Department \\ of Endocrinology, Università di Pisa, Pisa, Italy, ${ }^{3}$ Laboratory of Physiology and ${ }^{4}$ Service of Endocrinology of Erasme Hospital, Université Libre de Bruxelles, \\ Brussels, Belgium, ${ }^{5}$ Service of Endocrinology, CHR-Citadelle, Université de Liège, Liège, Belgium and ${ }^{6}$ Centre de Recherche en Neurosciences de Lyon, \\ Institut National de la Santé et de la Recherche Médicale U1028 - Centre National de la Recherche Scientifique UMR5292, Team 'WAKING - Integrative \\ Physiology of Brain Arousal Systems', Lyon, France
}

(Correspondence should be addressed to L L Morselli at Department of Medicine, Sleep, Metabolism and Health Center (SMAHC), MC 1027, University of Chicago; Email: Imorsell@medicine.bsd.uchicago.edu)

\begin{abstract}
Objectives: We previously reported that adult patients with GH deficiency (GHD) due to a confirmed or likely pituitary defect, compared with healthy controls individually matched for age, gender, and BMI, have more slow-wave sleep (SWS) and higher delta activity (a marker of SWS intensity). Here, we examined the impact of recombinant human $\mathrm{GH}(\mathrm{rhGH})$ therapy, compared with placebo, on objective sleep quality in a subset of patients from the same cohort.

Design: Single-blind, randomized, crossover design study.

Methods: Fourteen patients with untreated GHD of confirmed or likely pituitary origin, aged 22-74 years, participated in the study. Patients with associated hormonal deficiencies were on appropriate replacement therapy. Polygraphic sleep recordings, with bedtimes individually tailored to habitual sleep times, were performed after 4 months on rhGH or placebo.

Results: Valid data were obtained in 13 patients. At the end of the rhGH treatment period, patients had a shorter sleep period time than at the end of the placebo period $(479 \pm 11$ vs $431 \pm 19 \mathrm{~min}$ respectively; $P=0.005$ ), primarily due to an earlier wake-up time, and a decrease in the intensity of SWS (delta activity) $\left(559 \pm 125\right.$ vs $794 \pm 219 \mu \mathrm{V}^{2}$ respectively; $\left.P=0.048\right)$.

Conclusions: Four months of rhGH replacement therapy partly reversed sleep disturbances previously observed in untreated patients. The decrease in delta activity associated with rhGH treatment adds further evidence to the hypothesis that the excess of high-intensity SWS observed in untreated pituitary GHD patients is likely to result from overactivity of the hypothalamic GHRH system due to the lack of negative feedback inhibition by GH.
\end{abstract}

European Journal of Endocrinology 168 763-770

\section{Introduction}

A large body of evidence has demonstrated bidirectional interactions between somatotropic activity and sleep regulation (1). In particular, GHRH activity has been linked to the promotion of the deepest stages of nonrapid eye movement (NREM) sleep, i.e. slow-wave sleep (SWS) $(2,3,4,5)$. The absence of negative feedback regulation of $\mathrm{GHRH}$, as occurs in untreated $\mathrm{GH}$ deficiency (GHD) of pituitary origin, may result in a stimulation of the depth or intensity of NREM sleep, which can be estimated as EEG spectral power in the frequency band characteristic of slow waves (delta band: 0.75-4 Hz; also referred to as slow-wave activity (SWA) or delta activity).

Very few studies have objectively compared sleep quality in GHD patients and in appropriately matched controls and the results have been inconclusive $(6,7$, $8,9)$. We recently reported that sleep is disturbed in patients with GHD due to pituitary damage compared with individually age-, gender-, and BMI-matched controls. The disturbances involve excessive amounts of delta activity and an age-dependent increase in sleep fragmentation. Furthermore, the patients reported poor subjective sleep quality and daytime sleepiness, as well as impaired quality of life (QoL) (10).

Treatment of adult GHD patients with recombinant human GH (rhGH) is generally associated with a clear improvement in $\operatorname{QoL}(11,12,13)$. However, as far as sleep is concerned, the few studies that evaluated the effect of GH therapy did not detect any consistent change $(9,14,15,16,17)$. Therefore, the purpose of this study was to assess the impact of 4 months of rhGH treatment on sleep, compared with placebo, in adult 
GHD patients, using a single-blind, placebo-controlled, randomized, crossover design. We hypothesized that rhGH replacement would restore the negative feedback regulation of central GHRH activity and therefore correct the excessive SWA that we had observed in patients with pituitary GHD in the absence of treatment.

\section{Materials and methods}

\section{Patients and experimental protocol}

All 26 patients with GHD of confirmed or likely pituitary origin included in our study of sleep-wake regulation in untreated GH-deficient adults (10) were invited to participate in a single-blind, randomized, placebocontrolled, crossover design trial testing the impact of GH replacement therapy on sleep conducted at the Université Libre de Bruxelles (Belgium), the University of Pisa (Italy), the University of Liege (Belgium), and the University of Chicago (Illinois, USA). In each participating institution, the protocol was approved by the Institutional Review Board. Written informed consent was obtained from all participants.

Details of the inclusion criteria are given in our previous paper (10). In summary, GHD was diagnosed based on an i.v. insulin tolerance test performed within the last 5 years, with a maximum GH response $<3 \mu \mathrm{g} / \mathrm{l}$. Patients with evidence of substance abuse, liver disease, renal insufficiency, heart failure, malignant disease, chronic infectious disease, neurological or psychiatric disease, clinically significant hyperprolactinemia, or diabetes requiring administration of insulin or sulfonylureas were excluded from the study. Individuals employed as shift workers within the last 3 months and subjects having traveled across more than two time zones within 2 weeks before starting the study were not included. All subjects were off hypnotic drugs for at least 3 months. Additionally, patients were either rhGH naïve or had been off treatment for at least 6 months before participation in the study. All patients with associated pituitary hormonal deficiencies received stable replacement therapy, as assessed by at least two clinical and biological evaluations performed at intervals of at least 3 months.

Of the 26 patients with pituitary GHD included in our baseline study, six patients declined participation. Thus, 20 adults with pituitary GHD were enrolled. The patients were studied in the laboratories at the Universities of Chicago, Brussels (including five patients recruited at the University of Liège), and Pisa. The same procedures, equipment, instruments, and recording techniques were used at each site.

Immediately after completing the baseline study of sleep-wake regulation (10), patients underwent a first intervention period during which either rhGH or placebo was administered daily for 4 months. The dose of rhGH (Genotropin, Pharmacia) was sex and age
Table 1 Sex- and age-specific instructions for rhGH administration.

\begin{tabular}{lcccccc}
\hline & \multicolumn{2}{c}{ Men } & & \multicolumn{2}{c}{ Women } \\
\cline { 2 - 3 } \cline { 6 - 7 } & $\begin{array}{c}\leq 45 \\
\text { years }\end{array}$ & $\begin{array}{c}>45 \\
\text { years }\end{array}$ & & $\begin{array}{c}\leq 45 \\
\text { years }\end{array}$ & $\begin{array}{c}>45 \\
\text { years }\end{array}$ \\
\hline Initial dose (mg/day) & 0.2 & 0.1 & & 0.3 & 0.3 \\
Increments (mg/day) & 0.2 & 0.1 & & 0.2 & 0.1 \\
Maximum dose (mg/day) & 0.6 & 0.4 & & 0.7 & 0.6 \\
\hline
\end{tabular}

specific, according to the Consensus guidelines for the diagnosis and treatment of adults with GHD (18) (Table 1). Both Genotropin and placebo preparations were provided by Pharmacia. The dose of placebo was similarly titrated such that patients remained blind to the treatment condition. Patients were instructed to inject rhGH s.c., $\sim 30 \mathrm{~min}$ before bedtime. The participants returned for an outpatient visit at monthly intervals. Each visit included clinical examination, determination of IGF1 levels, and assessment (whenever applicable) of the current replacement therapy for associated pituitary hormonal deficiencies. The rhGH dosage was titrated accordingly by one of the physician investigators ( $\mathrm{L} \mathrm{L} \mathrm{M}$, J-J L, R E W, J M, or G C), who therefore did not remain blind to the nature of the injections. All other investigators and staff, including the sleep technologist who scored the recordings, were blind to the study condition.

At the end of this first 4-month period, the participants had an outpatient admission that included clinical examination and routine laboratory blood tests. Following this outpatient admission, the patients underwent 6 days of ambulatory sleep monitoring by wrist actigraphy (Actiwatch, Philips Respironics, Bend, OR, USA), a method providing accurate estimations of sleep onset and offset $(19,20)$. The mean habitual bedtimes from these recordings were used to individually design the bedtime schedule during a 2-day inpatient study, which occurred within 1 week after the end of ambulatory monitoring. The subjects were admitted to the laboratory between 1700 and $1900 \mathrm{~h}$ on day 1 and remained in the laboratory until discharge in the morning of day 3. Regular hospital meals were served at 0800,1230 , and $1900 \mathrm{~h}$. Lights were turned off $5 \mathrm{~min}$ before scheduled bedtime and turned on $5 \mathrm{~min}$ after scheduled wake time. During bedtimes, sleep was polygraphically recorded (DigiTrace Care Services, Boston, MA, USA).

Upon awakening on day 2, a blood sample was taken for measurement of plasma IGF1. Thereafter, patients were maintained under normal indoor light ( \pm 300 lux) until bedtime. During waking hours, they had sedentary activities (reading, watching TV, and simple neurobehavioral tests) and were free to ambulate around the unit. Naps were not allowed.

Immediately after this inpatient study, the participants entered a 3-month washout period followed by a 
second 4-month period during which either placebo or rhGH was administered. Objective sleep quality was reevaluated at the end of the second 4-month intervention period using the same procedures as during the first intervention period.

Throughout the study, patients who did not strictly conform to the instructions, who experienced side effects, or had to modify the replacement therapy for associated pituitary hormonal deficiencies were excluded from the study. At the end, valid sleep analyses in both placebo and rhGH conditions were obtained in 14 patients who completed the entire study. However, while reviewing the results of the spectral analysis of the sleep EEG, we noticed that one patient, a 29-year-old man who suffered a transection of the pituitary stalk (with associated hypoadrenalism, hypothyroidism, and hypogonadism), had results at the end of the placebo period that were widely divergent from his own recordings obtained during the baseline study (10), i.e. also in the absence of active treatment. His delta activity levels were indeed approximately seven times lower at the end of placebo treatment than at the end of baseline study. Delta activity is highly reproducible in a single individual $(21,22,23)$, even in recordings separated by several months, with intrasubject correlation coefficients above 0.90. The most likely explanation for the large between-study difference observed in this patient is a technical artifact related to amplifier settings. The Grubbs test for detection of statistical outliers $(24,25)$ performed on differences of both delta power and theta power during NREM sleep between the two recordings indeed identified this patient as a significant outlier $(P<0.01$ for delta power; $P<0.05$ for theta power), and he was excluded from the analysis. The results are therefore presented for 13 patients.

Four of them had childhood-onset idiopathic GHD that persisted into adulthood (as assessed by the persistence of an abnormally low $\mathrm{GH}$ response to the i.v. insulin tolerance test). Nine patients had an adult-onset GHD: in five of them, the origin of GHD was a primary pituitary defect without supra-pituitary involvement (surgical removal of a pituitary tumor without radiotherapy $(n=4))$. None of them presented with diabetes insipidus. One patient was diagnosed with pituitary apoplexy in a nonfunctioning pituitary adenoma and had diabetes insipidus. In two patients with adult-onset GHD, the existence of primary pituitary lesions was confirmed but a supra-pituitary involvement could not be excluded (surgical removal of a craniopharyngioma, without any adherence to the hypothalamus or the optic chiasm with the presence of diabetes insipidus $(n=2))$. Both pituitary and hypothalamic lesions were possible in one patient with histiocytosis and one patient with neurosarcoidosis. Individual patient descriptions are presented in Table 2.

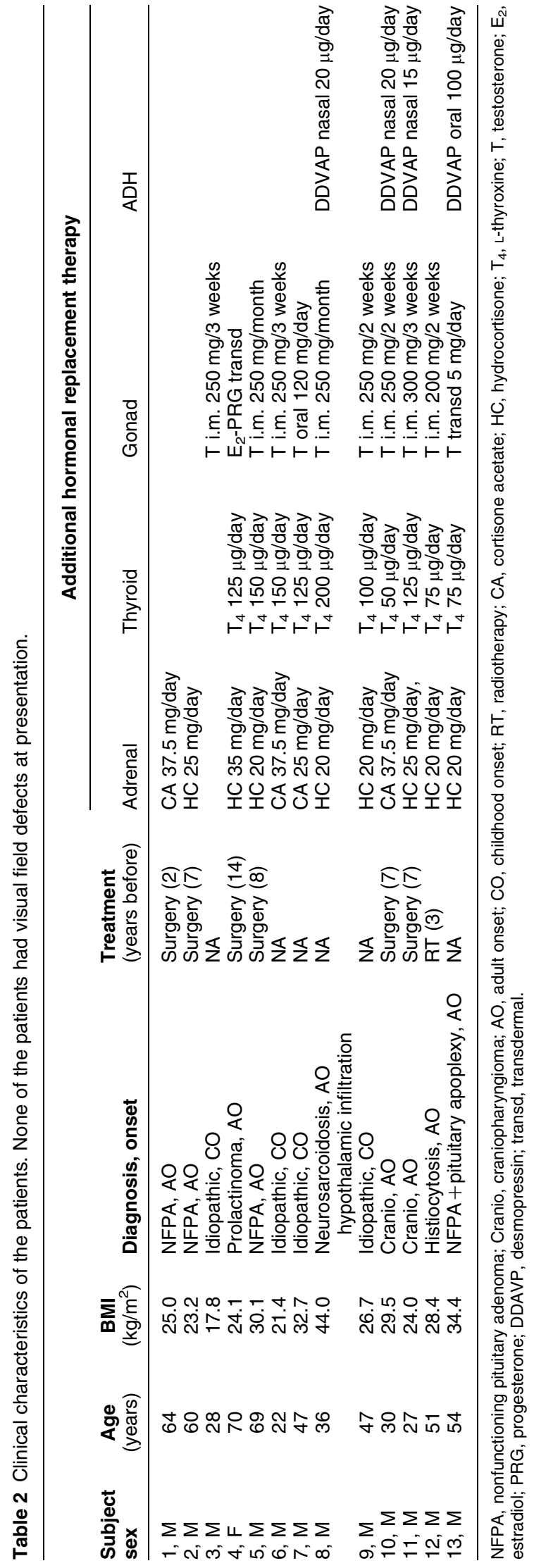

www.eje-online.org 


\section{Sleep analysis}

All polygraphic recordings were visually scored at 30-s intervals in stages wake, I, II, III, IV, and REM using standardized criteria (26) by the same experienced scorer who was blind to the subject's condition. Sleep onset and morning awakening were respectively defined as the times of the first and last 30-s intervals scored in stages II, III, IV, or REM. The sleep period was defined as the time separating sleep onset from final morning awakening. Total sleep time was defined as the sleep period minus the total duration of wake after sleep onset (WASO). Sleep latency was defined as the time from lights off until sleep onset. Sleep efficiency was calculated as the total sleep time expressed as percentage of the time allocated to sleep (i.e. the interval between lights off and lights on). Periodic leg movements were identified only in one recording in one patient.

A spectral analysis on the central EEG lead was performed (PRANA, PhiTools, Strasbourg, France). Muscular, ocular, and movement artifacts were eliminated before spectral analysis. Delta, theta, and alpha activities were calculated as the absolute spectral power in the frequency bands $0.75-4,4.5-8$, and $8.5-12 \mathrm{~Hz}$ respectively. Mean power per 30-s epoch was calculated for each band. Mean delta power in non-REM sleep quantifies the intensity or 'depth' of non-REM sleep. Alpha activity is an independent marker of the synchronization of high-frequency cortical oscillations that typically appear during quiet wake with eyes closed; it originates in neuronal networks distinct from those responsible for the generation of delta activity. Theta activity reflects spectral power in an intermediate frequency that is often contaminated by delta activity. For illustrative purposes, the durations of NREM/REM cycles were also normalized to account for individual differences, as described previously (27).

All the 56 nights recorded were scored; technical artifacts prevented spectral analysis for four of them. Comparisons between conditions used the second rather than the first night of polysomnography, because patients were habituated to the experimental procedures and spent the preceding day in the same standardized and controlled environment, except in four cases due to highly artifactual recordings.

\section{QoL and subjective sleep quality}

QoL was estimated by the global score of the QoL assessment of GHD in adults (QoL-AGHDA) questionnaire, which comprises 25 'yes or no' questions relative to specific complaints commonly reported by GHD patients (28). A higher score corresponds to lower QoL. Subjective sleep quality was assessed using the Pittsburgh Sleep Quality Index questionnaire (PSQI) (29). Additionally, the patients were asked to complete a Karolinska Sleep Log, a questionnaire used to assess subjective sleep duration and quality, for the six consecutive days preceding each inpatient session.

\section{Statistical analysis}

Comparisons between rhGH treatment and placebo were performed using the Wilcoxon signed rank test. All group values are expressed as means \pm s.E.M. All statistical calculations were performed using JMP version 8.0.2 (SAS Institute, Inc., Cary, NC, USA).

Table 3 Mean ( \pm S.E.M.) values of sleep variables in 13 patients with pituitary GHD after 4 months of placebo or rhGH treatment.

\begin{tabular}{lccc}
\hline & Placebo & GH & P level GH vs placebo \\
\hline Time of lights out & $2332 \mathrm{~h} \pm 12 \mathrm{~min}$ & $2344 \mathrm{~h} \pm 18 \mathrm{~min}$ & 0.470 \\
Time of lights on & $0754 \mathrm{~h} \pm 19 \mathrm{~min}$ & $0734 \mathrm{~h} \pm 20 \mathrm{~min}$ & 0.092 \\
Time in bed (min) & $504 \pm 13$ & $471 \pm 17$ & 0.216 \\
Sleep period time (min) & $479 \pm 11$ & $431 \pm 19$ & 0.005 \\
Sleep latency (min) & $15 \pm 4$ & $21 \pm 8$ & 0.423 \\
Total sleep time (min) & $422 \pm 20$ & $384 \pm 25$ & 0.223 \\
Sleep efficiency (\%) & $84 \pm 3$ & $82 \pm 4$ & 0.635 \\
WASO (min) & $57 \pm 16$ & $47 \pm 12$ & 0.191 \\
REM sleep (min) & $80 \pm 9$ & $67 \pm 8$ & 0.879 \\
REM sleep (\%) & $19 \pm 2$ & $17 \pm 2$ & 0.588 \\
Stages I+II (min) & $261 \pm 13$ & $248 \pm 15$ & 0.376 \\
Stages I+II (\%) & $63 \pm 4$ & $66 \pm 4$ & 0.542 \\
Stages III + IV (min) & $95 \pm 19$ & $69 \pm 19$ & 0.110 \\
Stages III+IV (\%) & $18 \pm 4$ & $17 \pm 4$ & 0.455 \\
Delta activity $\left(\mu \mathrm{V}^{2}\right)^{\mathrm{a}}$ & $794 \pm 219$ & $559 \pm 125$ & $\mathbf{0 . 0 4 8}$ \\
Theta activity $\left(\mu \mathrm{V}^{2}\right)^{\mathrm{a}}$ & $102 \pm 25$ & $82 \pm 17$ & 0.376 \\
Alpha activity $\left(\mu \mathrm{V}^{2}\right)^{\mathrm{a}}$ & $40 \pm 7$ & $35 \pm 5$ & 0.542 \\
\hline
\end{tabular}

WASO, wake after sleep onset; REM sleep, rapid eye movement sleep; NREM sleep, non-rapid eye movement sleep. aSpectral power is reported as the average absolute power during NREM sleep in the first $6 \mathrm{~h}$ of sleep.

Numbers in boldface indicate statistical significance and the number in italic indicates trend for significance. 


\section{Results}

Six patients started the study with the placebo phase and seven started with the rhGH phase. The average dose of $\mathrm{rhGH}$ was $0.48 \pm 0.03 \mathrm{mg} /$ day; the 'dose' of placebo averaged $0.50 \pm 0.07 \mathrm{mg} /$ day $(P=1.000$ vs GH). Plasma IGF1 levels averaged $231 \pm 20 \mathrm{ng} / \mathrm{ml}$ at the end of the $\mathrm{rhGH}$ period and $97 \pm 14 \mathrm{ng} / \mathrm{ml}$ at the end of the placebo period $(P<0.001)$ (age-adjusted SDSs: $-0.1 \pm 0.3$ s.D. vs $-2.1 \pm 0.2$ s.D. respectively; $P<0.001)$. BMI averaged $28.5 \pm 2.1 \mathrm{~kg} / \mathrm{m}^{2}$ at the end of the rhGH period vs $27.8 \pm 2.2 \mathrm{~kg} / \mathrm{m}^{2}$ at the end of the placebo period $(P=0.266)$.

\section{Objective sleep quality}

Table 3 summarizes the findings. The sleep period time (from sleep onset to final morning awakening) was reduced by an average of $48 \mathrm{~min}$ under rhGH compared with placebo $(P=0.005)$, primarily reflecting a trend to an earlier wake-up time. The relative distribution of the different sleep stages was not affected by treatment. The mean duration of SWS (stages III + IV) was reduced by $27 \%$, but this reduction did not reach statistical significance $(P=0.110)$. However, mean delta activity during NREM sleep in the first $6 \mathrm{~h}$ of sleep was decreased by $30 \%$ at the end of the rhGH treatment period, compared with the placebo period $(P=0.048)$ (Table 3). In contrast, theta and alpha activity levels were not affected by treatment. The average profiles of EEG delta, theta, and alpha power during the first four sleep cycles in the two conditions are presented in Fig. 1.

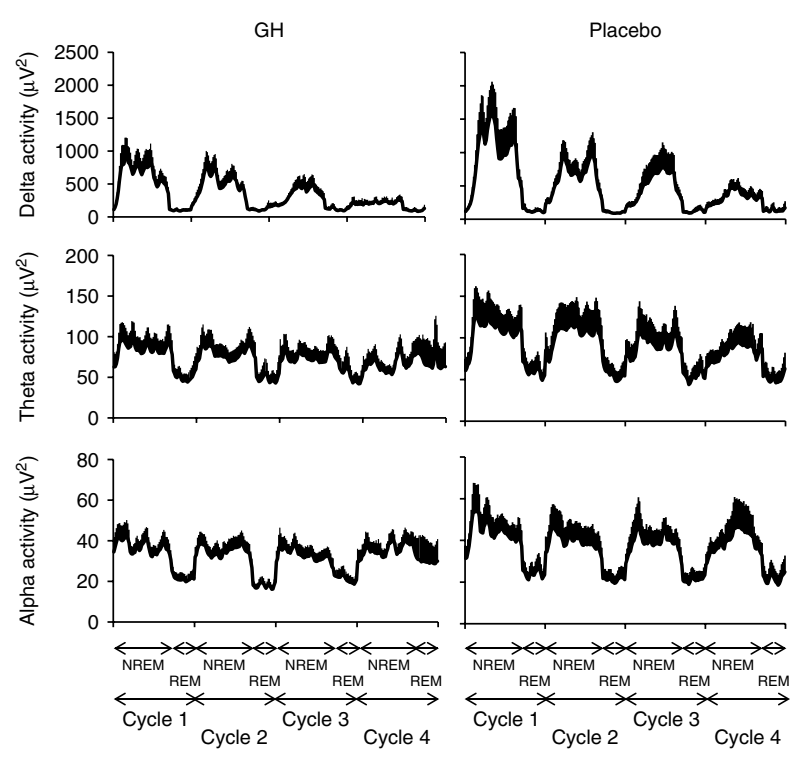

Figure 1 Mean profiles (+S.E.M.) of absolute EEG spectral power in the delta, theta, and alpha ranges during the first four NREM-REM cycles in GHD patients under rhGH (left) and placebo (right).
Of note, two patients displayed low sleep duration (at least $1.5 \mathrm{~h}$ less sleep in the laboratory than assessed at home by wrist actigraphy) as well as low sleep quality (average number of awakenings 46; average duration of WASO 2 h 40 min; average sleep efficiency 60\%) in both study conditions. If those two patients were excluded from the analysis, the reduction in the sleep period averaged $51 \mathrm{~min}(P=0.019)$, primarily reflecting earlier wake-up time $(P=0.027)$. The decrease in delta activity under rhGH treatment compared with placebo averaged $32 \% \quad(P=0.024)$. Results regarding sleep stages, theta and alpha activity levels remained similar in both conditions when these two patients were excluded.

\section{QoL and subjective sleep quality}

The global score of the QoL-AGHDA scale was decreased under $\mathrm{GH}$ compared with placebo $(4.75 \pm 0.57$ vs $8.75 \pm 0.89, P=0.023)$, indicating an improvement in QoL ratings. The PSQI averaged $4.1 \pm 0.7$ under rhGH vs $5.8 \pm 1.1$ under placebo, but this difference did not reach statistical significance $(P=0.102)$. Consistent with the polysomnographic findings, at the end of the $\mathrm{GH}$ treatment period, patients reported an earlier wake-up time (difference about $24 \mathrm{~min}, P=0.002$ ) and a shorter sleep time (difference about $46 \mathrm{~min}$, $P=0.007$ ) compared with the end of the placebo period.

\section{Discussion}

This study examined sleep duration, sleep architecture, and EEG power in the delta, theta, and alpha ranges in adult GHD patients of confirmed or likely pituitary origin, after 4 months of rhGH vs placebo administration. In light of the results of our previous study on adult patients with pituitary GHD (10), we hypothesized that the peculiar sleep disturbance observed in this cohort, i.e. an increase in NREM sleep intensity, would be reversed as the physiology of the somatotropic axis was restored by rhGH replacement. Compared with placebo, rhGH treatment in patients with pituitary GHD resulted in shorter sleep period time by almost $1 \mathrm{~h}$ and decreased intensity of NREM sleep as quantified by EEG delta activity. Additionally, in keeping with previous reports $(11,12,13)$, patients experienced an improvement in their QoL under rhGH.

A unique aspect of our study is that, in each treatment condition, the time allotted for sleep during the inpatient study was based on habitual sleep time derived from 1 week of ambulatory wrist actigraphy immediately preceding the inpatient study. Following rhGH treatment, the patients had shorter habitual time in bed than under placebo, primarily because they got out of bed earlier. Accordingly, sleep period time was nearly $1 \mathrm{~h}$ shorter under rhGH than under placebo. The shorter sleep period time could be explained by the 
lower levels of delta activity. Delta activity is a measure of sleep pressure and decreases exponentially throughout the night to reach minimal levels at the end of the night. This is thought to reflect the completion of the recovery process during sleep $(30,31)$. When delta activity is lower, the minimum is reached sooner and therefore the sleep episode is shorter. Vice versa, after a night of total sleep deprivation, a rebound of delta activity is typically observed during the following period of recovery sleep, which is typically longer than a regular night (32).

Sleep architecture (the proportions of the various sleep stages) was similar under both treatment conditions. The maintenance of a normal sleep architecture under rhGH treatment is in accordance with previous reports, as several authors observed no impact of replacement therapy administered for 6 months on sleep stages, compared with placebo (9) or to a baseline evaluation before treatment $(14,16,17)$ (Table 4). The only exception is a very small study $(n=5)$ that found a decrease in SWS duration in middle-aged patients with GHD of variable etiology after 6 months of rhGH withdrawal, compared with the results obtained after 1-2 years of high-dose rhGH replacement (15).

Quantitative EEG analysis of our results revealed a decrease in delta activity after rhGH replacement. In our previous study comparing sleep in patients with GHD of pituitary origin vs controls individually matched for age, gender, and BMI, we had observed excessive amounts of delta activity, associated with poor subjective sleep quality (10). We indicated that this combination of objective and subjective symptoms represents an unusual form of sleep disturbance as the most prevalent sleep disorders, such as obstructive sleep apnea, are characterized by the combination of reduced delta activity in association with increased daytime sleepiness $(33,34)$. While limited by the small sample size, the present findings nonetheless suggest that rhGH therapy corrects this peculiar sleep disturbance. Our results are consistent with the hypothesis of an enhanced GHRH drive in patients with GHD of pituitary origin, which could be reversed by rhGH treatment. Indeed, a large body of literature supports a role of GHRH in the modulation of sleep and in particular the generation of SWS/delta activity $(2,4,5)$. The only previous study (16) that had performed quantitative EEG analysis before and after rhGH treatment in GHD patients did not find any change in delta activity after 6 months of treatment, compared with baseline. No comparison was made with a placebo condition, and some patients experienced supraphysiological IGF1 concentrations with mild side effects.

Two recent studies, conducted in patients previously treated by transsphenoidal surgery for pituitary tumors with or without suprasellar extension, have evidenced that hypothalamic involvement was associated with sleep fragmentation, resulting in shorter sleep duration, disturbed sleep architecture, and disturbed circadian movement rhythms, possibly via alterations of the suprachiasmatic nuclei $(35,36)$. Though a

Table 4 Review of studies evaluating the impact of hGH treatment on sleep in adult GHD patients.

\begin{tabular}{|c|c|c|c|c|}
\hline Authors & $n$ & Patients & Protocol & Results \\
\hline \multirow[t]{2}{*}{$\begin{array}{l}\text { Aström et al. } \\
\text { (1990) (14) }\end{array}$} & 8 & $\begin{array}{l}\text { Young adults with } \\
\text { CO-isolated GHD }\end{array}$ & $\begin{array}{l}\text { PSG before and after } 6 \text { months of hGH } \\
\text { treatment }\end{array}$ & $\downarrow$ Total sleep time \\
\hline & & & $\begin{array}{l}\text { No placebo control } \\
\text { No spectral analysis }\end{array}$ & $\begin{array}{l}\uparrow \text { REM sleep } \\
\text { No change in SWS duration }\end{array}$ \\
\hline $\begin{array}{l}\text { Nolte et al. } \\
(2002)(15)\end{array}$ & 5 & $\begin{array}{l}\text { Patients with AO-GHD } \\
\text { due to pituitary disease }\end{array}$ & $\begin{array}{l}\text { PSG after } 1 \text { year rhGH treatment and } \\
\text { after } 6 \text { months of withdrawal } \\
\text { No placebo control } \\
\text { No spectral analysis }\end{array}$ & $\begin{array}{l}\text { rhGH: normal stages I, II, SWS duration; } \\
\downarrow \text { sleep efficiency and REM sleep } \\
\text { rhGH withdrawal: } \downarrow \text { SWS duration }\end{array}$ \\
\hline \multirow[t]{2}{*}{$\begin{array}{l}\text { Schneider et al. } \\
\text { (2005) (16) }\end{array}$} & 18 & CO-GHD patients & $\begin{array}{l}\text { PSG before and after } 6 \text { months of rhGH } \\
\text { treatment. No placebo control }\end{array}$ & No differences in sleep parameters \\
\hline & & & Spectral analysis & $\begin{array}{l}\text { Supraphysiological IGF1 levels in ten } \\
\text { patients }\end{array}$ \\
\hline $\begin{array}{l}\text { Peker et al. } \\
(2006)(17)\end{array}$ & 19 & AO-GHD patients & $\begin{array}{l}\text { PSG before and after } 6 \text { months of rhGH } \\
\text { treatment } \\
\text { No placebo control } \\
\text { No spectral analysis }\end{array}$ & No differences in sleep parameters \\
\hline $\begin{array}{l}\text { Ismailogullari } \\
\text { et al. (2009) (9) }\end{array}$ & 12 & $\begin{array}{l}\text { Women with AO-GHD } \\
\text { due to Sheehan's } \\
\text { syndrome }\end{array}$ & $\begin{array}{l}\text { Parallel group design PSG before and } \\
\text { after } 6 \text { months of rhGH treatment and } \\
\text { vs eight patients on placebo } \\
\text { No spectral analysis }\end{array}$ & No differences in sleep parameters \\
\hline \multirow[t]{2}{*}{$\begin{array}{l}\text { Morselli et al. } \\
\text { (present study) }\end{array}$} & 13 & $\begin{array}{l}\text { Nine adults with } \\
\text { AO-GHD }\end{array}$ & $\begin{array}{l}\text { Randomized crossover design PSG after } \\
4 \text { months of treatment with rhGH vs } \\
\text { placebo }\end{array}$ & $\begin{array}{l}\text { rhGH vs placebo: } \downarrow \text { sleep period time } \\
\quad \text { (earlier wake up) }\end{array}$ \\
\hline & & $\begin{array}{l}\text { Four adults with } \\
\text { CO-GHD }\end{array}$ & Spectral analysis & $\downarrow$ SWS intensity (delta activity) \\
\hline
\end{tabular}

CO, childhood onset; AO, adult onset; PSG, polysomnography; REM, rapid eye movement; SWS, slow-wave sleep. 
hypothalamic involvement could not be excluded in four of our patients, none had suffered from compression of the optic chiasm and only one had received radiotherapy. Moreover, sleep variables were similar in those patients and in the other nine patients (data not shown). Thus, it seems unlikely that hypothalamic alterations were involved in sleep disturbances observed in our cohort.

Our study has several limitations. The small sample size is an obvious weakness of the study, which involved a demanding and labor-intensive protocol and challenging recruitment criteria. Secondly, our cohort mostly consisted of middle-aged and older men (8/13 patients over 40 years old). In men, the earliest sign of aging of sleep is a dramatic decrease in delta activity that is apparent already by 40 years of age $(37,38)$. Therefore, it is possible that the impact of rGH treatment on sleep, and particularly SWS and delta activity, would have been more robust if we had been able to recruit more young patients. A further limitation is that we did not record breathing parameters in these patients and therefore could not control for the presence of sleep apnea. An increase in the prevalence and severity of sleep apnea could have contributed to the decrease in delta activity observed in our study. However, neither the distribution of sleep stages nor the measures of sleep fragmentation (which would arguably increase in the case of worsening sleep apnea) were affected by treatment. The fact that the participants in this study were only modestly overweight and did not gain a significant amount of weight during rGH treatment also argues against a role of sleep apnea in the present findings. Finally, the only prospective study evaluating the incidence of sleep apnea in adult GHD patients treated with rhGH showed that treatment does not induce or aggravate sleep apnea (17).

\section{Conclusion}

In conclusion, although they need to be confirmed by larger studies, the present findings suggest that rhGH treatment administered for 4 months may reverse some of the disturbances in the macro- and micro-architecture of sleep previously evidenced in adult patients with GHD. These findings add to a body of evidence indicating a central role of GHRH activity in modulating sleep quality.

\section{Declaration of interest}

The authors declare that there is no conflict of interest that could be perceived as prejudicing the impartiality of the research reported.

\section{Funding}

This study was sponsored by an investigator-initiated grant from Pharmacia Corporation and received partial support from NIH grant MO1 RR-00055 to the General Clinical Research Center of the
University of Chicago. During part of the study, Dr A Nedeltcheva was supported by the Northwestern University - University of Chicago Training Grant for Sleep Research (NIH grant T32 HL-07909). The analysis of sleep recordings was partly supported by grant PO1 AG-11412 from the National Institute on Aging.

\section{Author contribution statement}

All authors in this manuscript have contributed substantially to the manuscript, either in design, data collection, or analysis and drafting of the article and have provided final approval before submission of the manuscript.

\section{Acknowledgements}

The authors thank Claire van den Bril, MS (Université Libre de Bruxelles), Elisabeth Lallemant, MS (CHR-Citadelle, Liège), and the nursing staff of the General Clinical Research Center of the University of Chicago for excellent care of the subjects during inpatient studies. They are also grateful to Dr P Penev for assistance with the screening of subjects recruited at the University of Chicago and to Dr Barbara Lippe (formerly of Pharmacia Corporation) for helpful advice and suggestions during the development of the protocol.

\section{References}

1 Van Cauter E, Plat L \& Copinschi G. Interrelations between sleep and the somatotropic axis. Sleep 199821 553-566.

2 Steiger A, Guldner J, Hemmeter U, Rothe B, Wiedemann K \& Holsboer F. Effects of growth hormone-releasing hormone and somatostatin on sleep EEG and nocturnal hormone secretion in male controls. Neuroendocrinology 199256 566-573. (doi:10.1159/ 000126275)

3 Kerkhofs M, Van Cauter E, Van Onderbergen A, Caufriez A, Thorner MO \& Copinschi G. Sleep-promoting effects of growth hormone-releasing hormone in normal men. American Journal of Physiology. Endocrinology and Metabolism 1993264 E594-E598.

4 Obal F Jr \& Krueger JM. GHRH and sleep. Sleep Medicine Reviews 20048 367-377. (doi:10.1016/j.smrv.2004.03.005)

5 Peterfi Z, McGinty D, Sarai E \& Szymusiak R. Growth hormonereleasing hormone activates sleep regulatory neurons of the rat preoptic hypothalamus. American Journal of Physiology. Regulatory, Integrative and Comparative Physiology 2010298 R147-R156. (doi:10.1152/ajpregu.00494.2009)

6 Orr W, Vogel G, Stahl M, Griffiths W \& Seely J. Sleep patterns in growth hormone deficient children and age-matched controls: developmental considerations. Neuroendocrinology 1977 24 347-352. (doi:10.1159/000122721)

$7 \mathrm{Wu}$ RH \& Thorpy MJ. Effect of growth hormone treatment on sleep EEGs in growth hormone-deficient children. Sleep 198811 $425-429$.

8 Aström C \& Jochumsen PL. Decrease in delta sleep in growth hormone deficiency assessed by a new power spectrum analysis. Sleep 198912 508-515.

9 Ismailogullari S, Tanriverdi F, Kelestimur F \& Aksu M. Sleep architecture in Sheehan's syndrome before and 6 months after growth hormone replacement therapy. Psychoneuroendocrinology 200934 212-219. (doi:10.1016/j.psyneuen.2008.09.002)

10 Copinschi G, Nedeltcheva A, Leproult R, Morselli LL, Spiegel K, Martino E, Legros JJ, Weiss RE, Mockel J \& Van Cauter E. Sleep disturbances, daytime sleepiness, and quality of life in adults with growth hormone deficiency. Journal of Clinical Endocrinology and Metabolism 201095 2195. (doi:10.1210/jc.2009-2080)

11 Koltowska-Haggstrom M, Mattsson AF, Monson JP, Kind P, Badia X, Casanueva FF, Busschbach J, Koppeschaar HP \& Johannsson G. Does long-term GH replacement therapy in 
hypopituitary adults with GH deficiency normalise quality of life? European Journal of Endocrinology $2006 \quad 155$ 109-119. (doi:10.1530/eje.1.02176)

12 Woodhouse LJ, Mukherjee A, Shalet SM \& Ezzat S. The influence of growth hormone status on physical impairments, functional limitations, and health-related quality of life in adults. Endocrine Reviews 200627 287-317. (doi:10.1210/er.2004-0022)

13 Moock J, Albrecht C, Friedrich N, Volzke H, Nauck M, KoltowskaHaggstrom M, Kohlmann T \& Wallaschofski H. Health-related quality of life and IGF-1 in GH-deficient adult patients on GH replacement therapy: analysis of the German KIMS data and the Study of Health in Pomerania. European Journal of Endocrinology 2009160 17-24. (doi:10.1530/EJE-08-0738)

14 Aström C, Pedersen SA \& Lindholm J. The influence of growth hormone on sleep in adults with growth hormone deficiency. Clinical Endocrinology 199033 495-500. (doi:10.1111/j.13652265.1990.tb03886.x)

15 Nolte W, Radisch C, Rodenbeck A, Wiltfang J \& Hufner M. Polysomnographic findings in five adult patients with pituitary insufficiency before and after cessation of human growth hormone replacement therapy. Clinical Endocrinology 200256 805-810. (doi:10.1046/j.1365-2265.2002.01531.x)

16 Schneider HJ, Oertel H, Murck H, Pollmacher T, Stalla GK \& Steiger A. Night sleep EEG and daytime sleep propensity in adult hypopituitary patients with growth hormone deficiency before and after six months of growth hormone replacement. Psychoneuroendocrinology 200530 29-37. (doi:10.1016/j.psyneuen.2004.05.004)

17 Peker Y, Svensson J, Hedner J, Grote L \& Johannsson G. Sleep apnoea and quality of life in growth hormone (GH)-deficient adults before and after 6 months of GH replacement therapy. Clinical Endocrinology $2006 \quad 65$ 98-105. (doi:10.1111/j.1365-2265. 2006.02555.x)

18 Ho KK. Consensus guidelines for the diagnosis and treatment of adults with GH deficiency II: a statement of the GH Research Society in association with the European Society for Pediatric Endocrinology, Lawson Wilkins Society, European Society of Endocrinology, Japan Endocrine Society, and Endocrine Society of Australia. European Journal of Endocrinology 2007157 695-700. (doi:10.1530/EJE-07-0631)

19 Jean-Louis G, von Gizycki H, Zizi F, Spielman A, Hauri P \& Taub H. The actigraph data analysis software: I. A novel approach to scoring and interpreting sleep-wake activity. Perceptual and Motor Skills 199785 207-216. (doi:10.2466/pms.1997.85.1.207)

20 Jean-Louis G, von Gizycki H, Zizi F, Spielman A, Hauri P \& Taub H. The actigraph data analysis software: II. A novel approach to scoring and interpreting sleep-wake activity. Perceptual and Motor Skills 199785 219-226. (doi:10.2466/pms.1997.85.1.219)

21 Tan X, Campbell IG, Palagini L \& Feinberg I. High internight reliability of computer-measured NREM delta, sigma, and beta: biological implications. Biological Psychiatry $2000 \quad \mathbf{4 8}$ 1010-1019. (doi:10.1016/S0006-3223(00)00873-8)

22 Tan X, Campbell IG \& Feinberg I. Internight reliability and benchmark values for computer analyses of non-rapid eye movement (NREM) and REM EEG in normal young adult and elderly subjects. Clinical Neurophysiology 2001112 1540-1552. (doi:10.1016/S1388-2457(01)00570-3)

23 Tasali E, Spiegel K, Latta F, Leproult R \& Van Cauter E. Slow wave activity is a stable trait dependent characteristic in both young and older adults. In Society for Research on Biological Rhythms Annual meeting, Amelia Island, FL, May 22-26, 2002, 2002.

24 Grubbs FE. Procedures for detecting outlying observations in samples. Technometrics 196911 1-21. (doi:10.1080/00401706. 1969.10490657)
25 Grubbs FE \& Beck G. Extension of sample sizes and percentage points for significance tests of outlying observations. Technometrics 197214 847-854. (doi:10.1080/00401706.1972.10488981)

26 Rechtschaffen A \& Kales A. A Manual of Standardized Terminology, Techniques and Scoring System for Sleep Stages of Human Subjects. UCLA Brain Information Service/Brain Research Institute, Los Angeles, 1968.

27 Landolt HP. Sleep homeostasis: a role for adenosine in humans? Biochemical Pharmacology 200875 2070-2079. (doi:10.1016/j. bcp.2008.02.024)

28 McKenna SP, Doward LC, Alonso J, Kohlmann T, Niero M, Prieto L \& Wiren L. The QoL-AGHDA: an instrument for the assessment of quality of life in adults with growth hormone deficiency. Quality of Life Research 19998 373-383. (doi:10.1023/ A:1008987922774)

29 Buysse DJ, Reynolds CF III, Monk TH, Berman SB \& Kupfer DJ. The Pittsburgh sleep quality index: a new instrument for psychiatric practice and research. Psychiatry Research 198928 193-213. (doi:10.1016/0165-1781(89)90047-4)

30 Borbely AA. A two process model of sleep regulation. Human Neurobiology 19821 195-204.

31 Daan S, Beersma DG \& Borbely AA. Timing of human sleep: recovery process gated by a circadian pacemaker. American Journal of Physiology. Endocrinology and Metabolism 1984246 R161-R183.

32 Dijk D, Brunner DP \& Borbely AA. Time course of EEG power density during long sleep in humans. American Journal of Physiology. Regulatory, Integrative and Comparative Physiology 1990258 R650-R661.

33 Heinzer R, Gaudreau H, Décary A, Sforza E, Petit D, Morisson F \& Montplaisir J. Slow-wave activity in sleep apnea patients before and after continuous positive airway pressure treatment. Chest 2001119 1807-1813. (doi:10.1378/chest.119.6.1807)

34 Moyer CA, Sonnad SS, Garetz SL, Helman JI \& Chervin RD. Quality of life in obstructive sleep apnea: a systematic review of the literature. Sleep Medicine 20012 477-491. (doi:10.1016/S13899457(01)00072-7)

35 Borgers AJ, Romeijn N, van Someren E, Fliers E, Alkemade A \& Bisschop PH. Compression of the optic chiasm is associated with permanent shorter sleep duration in patients with pituitary insufficiency. Clinical Endocrinology $2011 \quad \mathbf{7 5} \quad 347-353$. (doi:10.1111/j.1365-2265.2011.04053.x)

36 Biermasz N, Joustra S, Donga E, Pereira A, van Duinen N, van Dijk M, van der Klaauw A, Corssmit E, Lammers G \& van Kralingen K. Patients previously treated for nonfunctioning pituitary macroadenomas have disturbed sleep characteristics, circadian movement rhythm, and subjective sleep quality. Journal of Clinical Endocrinology and Metabolism 201196 1524-1532. (doi:10.1210/jc.2010-2742)

37 Ehlers CL \& Kupfer DJ. Slow-wave sleep: do young adult men and women age differently? Journal of Sleep Research 19976 211-215. (doi:10.1046/j.1365-2869.1997.00041.x)

38 Van Cauter E, Leproult R \& Plat L. Age-related changes in slow wave sleep and REM sleep and relationship with growth hormone and cortisol levels in healthy men. Journal of the American Medical Association 2000284 861-868. (doi:10.1001/jama.284.7.861)

Received 27 November 2012

Revised version received 7 February 2013

Accepted 27 February 2013 